http://jmscr.igmpublication.org/home/

ISSN (e)-2347-176x ISSN (p) 2455-0450

crossref DOI: https://dx.doi.org/10.18535/jmscr/v7i7.142

Journal Of Medical Science And Clinical Research

IGM Publication

An Official Publication of IGM Publication

\title{
Study of Weekly Supervised Home Based Developmental Activity Program for Children with Global Developmental Delay
}

\author{
Author \\ Pankaj M Buch \\ Professor of Pediatrics, M.P.Shah Government Medical College and G.G.G. Hospital, Jamnagar, \\ Gujarat India Pin: 361008 \\ *Corresponding Author \\ Dr Pankaj M Buch \\ Department of Pediatrics, M.P.Shah Government Medical College, Jamnagar, Gujarat India
}

\begin{abstract}
Background: Developmental delay is common health problem that affect pediatric population. Global developmental delay (GDD) is defined as delay in two or more domains of development. Developmental assessment scale for Indian infants (DASII) is valid and reliable tool for developmental assessment up to 30 months of age. Early intervention is crucial for better outcome in GDD. There is a need for supervised home program for intervention for developmental delay to make it more feasible and to have better outcome.

Methods: study was conducted in District early Intervention centre (DEIC). Sociodemographic profile and baseline DASII score was obtained prior to study. Parents were trained in home program based. Weekly institute follow up was ensured for monitoring of home therapy. After six month of regular follow up DASII was repeated and difference in means of motor and mental quotients were noted. Correlation analysis was done between significant variables.

Results: Total of 52 children was enrolled in study. Age range was 3-24 months. Male to female ratio was 1.7:1. Fifty-Eight percentages had having mild developmental delay, $31 \%$ had moderate delay where as 7\% had severe and 4\% profound developmental delay. There was significant difference between pre-and post therapy DASII scores in motor and mental scale with large effect size (Cohen's $d$ $>0.7$ ). children across all severity of GDD showed improvement from home based therapy supervised by weekly institute visits.

Conclusions: Early intervention program are helpful for management of GDD. Addition of structured and supervised home activity program may augment developmental progression.

Keywords: Global Developmental delay, DASII, Early intervention.
\end{abstract}

\section{Introduction}

Development during the early childhood, especially from fetus to two-year-old (the first 1,000 days since life starts), is crucial in determining the nutrition and health status in the whole life course. Brain and nervous system development begins early in pregnancy and is largely complete by the time the child reaches the age of $2^{[1]}$.

Developmental delays are common health problem that affect $5-10 \%$ of general pediatric population. Domains of development are gross 
motor, fine motor-adaptive, persono socialactivities of daily living (ADL) and language Developmental delay occurs either in single domain such as motor or language or may present as Global Developmental Delay (GDD). GDD can be defined as developmental delay in two or more domains. Developmental delay can be classified as motor delay, cerebral palsy, isolated language delay, intellectual disability/ GDD, and pervasive developmental delay or profound sensory impairments ${ }^{([2],[3])}$.

There has been a significant decline in mortality among young children in low- and middle income countries (LMICs), largely due to world campaigns to eliminate or reduce the impact of fatal childhood diseases ${ }^{[4]}$. Better perinatal care, early identification and appropriate and timely referral has contributed a lot in reducing neonatal and infant mortality in developing countries. Studies of survivors of the diseases, however, show a relatively high prevalence of neurodevelopmental delays. Thus there is an emerging population of children, estimated at over 200 million, with developmental delays and disabilities in LMICs. Intellectual/developmental disabilities often lead to emotional and financial impacts on individuals and families and their quality of life $\mathrm{e}^{[5]}$.

A systematic review from the United States reported a prevalence of developmental delay of $0.66 \%$ in children and adolescents in $2012^{[6]}$. There is paucity of community-based data from developing countries, but it stands to reason that the prevalence would be similar, if not considerably higher. The main reason for this is the logistic challenges that arise in establishing diagnosis in the community using valid and reliable tools. For instance, in India, the prevalence of individuals with Mental Retardation according to the 2011 census is reported as $5.6 \%$, but further age stratification is unavailable.

Determining etiology is also a very challenging task especially in resource poor set up. There is need of more medical professionals (physicians, pediatricians, neurologists, pathologists, psychologists and psychiatrists) to get involved in management of GDD/MR; more funds and advanced tests (genetic, biochemical and neuropath logical) should become available for research; and GDD/ Mental Retardation should not be considered to be an isolated medical problem, but one in which the psychological, psychiatric, educational and sociological aspects should be considered concurrently ${ }^{[7]}$.

Though in many cases etiology of GDD/ID remains elusive, management of GDD is straightforward. Therapy is imparted based on assessment of current level of functioning which is followed by deciding short term and long term goals. Each goal is divided in to various tasks. In therapy session these tasks are taught/ practiced by reinforcement methods for mental/cognitive domains and by various physical activities / physiotherapy for motor domains. In the Western/developed countries, parent participation has been documented as playing an important role in fostering optimal development for children with disabilities $^{([8],[9])}$. In particular, a home-based intervention that includes parent training and involvement is an effective approach for early intervention that aims at improving parent-child outcomes. The family environment is a natural setting in which services can be delivered to foster mutual enjoyment, parent skills, and the child's social and cognitive competence. A number of studies suggest that home-based interventions improve child and family outcomes ([10],[11],[12]).

One such home based intervention project is the Portage project. It was originally created to provide home-based services in rural communities to young children with disabilities in the U.S. ${ }^{[13]}$. It has been widely adopted internationally, especially in developing countries, and has been translated into 36 languages $^{[14]}$. It has been an effective program in training parents to work with their children where there are no professional resources available .Its advantages include the availability of a ready-made curriculum, assessment materials and instruction manual. The curriculum is easy to learn and can be used by 
paraprofessionals. The developmental areas targeted by the Portage curriculum for preschoolers are motor, language, cognitive and social from birth to six years age $\mathrm{e}^{[15]}$.

In India, National Institute of Mental Handicap (NIMH) has also developed manuals that can be used by therapist and/or parents for intervention for developmental delays. These simple activities are easy to perform, it requires less resources and can yield better outcome. ${ }^{([16],[17],[18])}$

Under National Health Mission Government of India and then state government has established District Early Intervention Centre (DEIC). Main function of DEIC is to provide preventive, promotive, curative and referral services to children with developmental delay/ disabilities. As per experience of author there is discrepancy between available strength of therapist and patient load in DEIC. Majority of Indian population lives in rural areas so additional home based program supervised by scheduled visits at therapy centre may bring better results. It also reduces economic burden on family to bring child daily at centre. Daily or too frequent visits for therapy at intervention centre may also lead to poor adherence to therapy as developmental delay requires long term therapy and it imparts strain on family dynamics. Home based therapy supervised with weekly visit can be an alternative for same if not better outcome.

Present study was planned to see the impact of combination of weekly clinic based plus daily home based therapy for children with global developmental delay.

\section{Methods}

This prospective interventional study was undertaken at District Early Intervention Centre (DEIC) attached to medical college level government hospital. DEIC works in a multidisciplinary manner and is referral unit for developmental disabilities for surrounding districts. After institutional ethical clearance study was conducted for the period from December 2016 to June 2018.
Inclusion criteria for the present study were, (1) children in the age range of 0-30 months at time of enrollment (2) children with a diagnosis of global developmental delay. (3) Regular once a week follow up to DEIC for 6 months.

Exclusion criteria: (1) prior therapy for developmental delay. (2) Children with congenital malformations, isolated language delay, autism spectrum disorder, muscular dystrophy, congenital myopathy or other neuromuscular disorders.(3) children who were absent for more than 2 consecutive visits any time in six month therapy period.

The demographic characteristics of children and their families were collected in form of age, sex, socioeconomic status, habitat and, education level of parents. Relevant clinical history and anthropometry assessment was done.

Developmental assessment was done by Developmental Assessment Scale for Indian Infants (DASII) by a trained psychologist having prior exposure to test for more than 3 years. DASII is a diagnostic norm referenced test. It has point scale with items arranged in ascending order of age placement for both motor as well as mental scales. DASII has motor scale comprising of 67 items and mental scale comprising of 163 items. Both the scales have been divided in different clusters. Motor clusters comprises of neck control, body control, coordinated movements, locomotion skills and manipulation. Mental clusters comprises of visual and auditory cognizance, reaching, manipulation, memory, social interaction and imitative behavior, language, understanding of relationship, differentiation and manual dexterity. Motor and mental Developmental quotients (MoDQ and MeDQ) are calculated from DASII and DQ less than 70 was considered as significant [19]. Categorization of GDD was done as per IQ based guidelines of mental retardation applied in similar study from India ${ }^{[20]}$.

After first/ baseline assessment, family members of the children with developmental delay were explained about importance of regular intervention activity at home and follow up at 
institute. As per baseline level of a particular child, long term and short term goals were planned. Activities to achieve that particular goal were based on of NIMH, Secunderabad modules and portage community based rehabilitation module ${ }^{([13],[16],[17],[18])}$. Author has got trained in implementation of portage module from CBR network, Bangalore, India. Team of consultant and therapist in form of pediatrician, special educator, physiotherapist and social worker imparted training to family members usually mother or grandmother to carry out activities at home. Every family was given a notebook in which they were given complete details about home activity. Special education /early intervention, occupational and simple physiotherapy activities were demonstrated to them in a session of half an hour.This demonstration was followed by recall from them in form of they were suppose to practice in presence of therapist. Feedback was given for improvement as needed. Parents were also allowed to video shoot important elements of training so that they can refer it at home along with written instruction as and when needed.

Children with GDD were allotted a specific time for follow up. Follow up sheet was created to check for adherence to therapy. If the parents miss two follow up visits or three visits total they were telephonically called and adherence was complied upon. If child was absent for more than 3 visits, he /she was dropped from study. When a particular task/short term goal was achieved next higher goal was demonstrated to be practiced at home.

At the end of six month of therapy again developmental level of each child was assessed by DASII. The differences if any in scores of motor and mental scale of DASII were noted down. Enough care was taken to prevent bias as at the time of $2^{\text {nd }}$ DASII assessment at 6 month, as psychologist did not have access to baseline DASII scores. Data was compiled and analysis was done by Epi info version 7 and by SOFA software. Means or pre and post therapy (baseline versus six month follow up) were compared by paired $t$ test. $P$ value less than 0.05 was considered significant. We calculated the effect size for each intervention using Cohen's $\mathrm{d}$, defined as the difference between the means of the treatment and control groups divided by the pooled standard deviation (SD) of the population from which the groups were sampled Effect size was calculated for paired sample. Effect size of 0.5 was considered as moderate and more than 0.7 was considered as large effect size. Spearmen's correlation was used to find any relation between important variables like age of starting therapy, type of family, duration of home therapy and educational level of family and improvement in developmental scores.

\section{Results}

In present study 133 children who met inclusion criteria were enrolled but only 52 children could complete six month centre+ home therapy sessions with predecided follow up visits. Mean age of starting therapy was $12.4 \pm 8.6$ months with age range of 3-24 months. Median age was 9 month. Study comprised of 33 male children and 19 female children with male to female ratio of 1.73:1.

Sixty-five percentage of families belonged to urban set up and $60 \%$ of families belonged to joint family. Uneducated father accounted for $13 \%$ where as $20 \%$ of mother were uneducated. Majority of parents had education up to secondary schooling.

Categorization of GDD among children was suggestive of $30 / 52(58 \%)$ having mild developmental delay, 16/52(31\%) had moderate delay where as $04 / 52(7 \%)$ and $2 / 52(4 \%)$ had severe and profound developmental delay consecutively.

Home therapy was given for 30 minutes in $38 / 52$ (73\%) children and between 30- 60 minutes was given in 14/52(27\%) children. In all the cases home therapy was given by mother and supported partly by grandmother in a case of joint family. 
Figure 1 demonstrated difference in mean \pm SD at baseline and six month follow up DASII score in motor and mental scales.

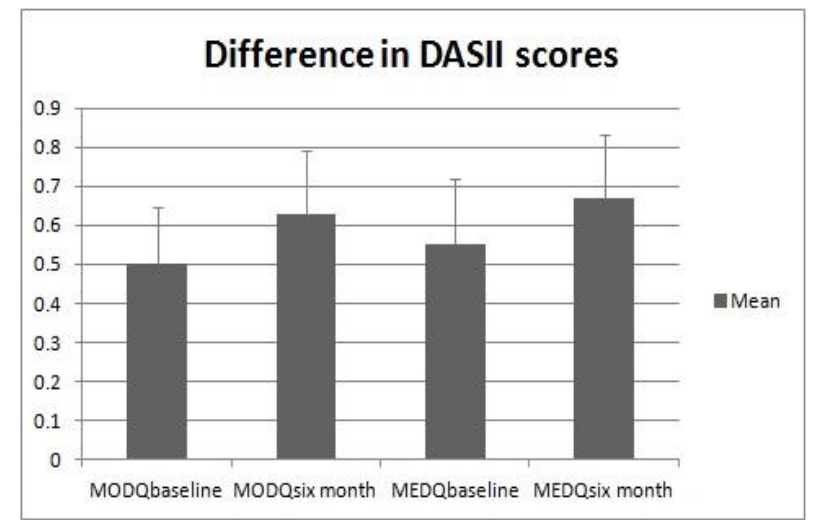

Figure 1: Difference in means of DASII score before and after therapy

In present study correlation was found between age of starting therapy and severity of GDD $(\mathrm{p}=0.02, \mathrm{R}$ stat 0.3$)$ as well as with gender of a child ( $\mathrm{p}=0.04$, $\mathrm{R}$ stat 0.283 ). No correlation was found between age of starting therapy or education of parents with difference in motor and mental DQ at 6 months of therapy. It was found that mild correlation existed between place of residence $(\mathrm{p}<0.04, \mathrm{R}$ stat 0.28$)$ and age of staring therapy.

Modest correlation was found between family type and improvement in mental DQ at 6 months of age $(p<0.001, R$ stat 0.444$)$. Children living in joint family environment had better improvement in mental score at 6 months of therapy however in present study, correlation was not found between type of family and improvement in motor development at 6-month therapy. There was no significant correlation between severity of GDD and duration of home therapy or score of developmental quotient at 6 month of therapy.

In present study large effect size were noted between scores in motor and mental developmental quotients at baseline (start of therapy) and at six months follow up. Effect size (Cohen's d) was 0.784 for mental developmental quotient and 0.938 for motor development quotient.

\section{Discussion}

In present study though 133 children with global developmental delay were enrolled to begin with, only 52 children (39\%) could complete the study. In a study by Lakhan $\mathrm{R}$ et $\mathrm{al}^{[20]}$ they could enroll 67 children from 63 villages of a particular block from India who were adherent to therapy. In a study by Mei.-Hua Tang et al in Taiwan ${ }^{[2]}$ they had to exclude $18 \%$ of children because of poor adherence and other factors. Adherence to therapy is very critical but at times most neglected part by the family members looking to longer duration and possible lifelong impairment. Bringing frequently child with global developmental delay to clinic poses many logistic issues especially in resource constrained family environment. Educational level of parents, socioeconomic structure and distance of therapy centre from residence would have contributed to this finding and differences in present study.

Male to female ratio in present study of $1.73: 1$ is comparable to study by Mei. -Hua Tang et al ${ }^{[2]}$ having ratio of 1.8:1 in their study in their study from northern India showed male to female ratio of 2.4:1. Lakhan $\mathrm{R}$ et $\mathrm{al}^{[20]}$ in their study from tribal part of India showed male to female ratio of 1.3:1. Such variation is possible because of place of study such as community settings or clinic based and socioeducational and cultural profile of particular region. As mentioned by study from Paramleen Kaur et al $^{[21]}$ Higher percentage of males in the population may be explained by the tendency of the families to nurture a male child more as compared to the female child expecting financial contributions from the male children once they grow up.

Different studies about addition of home based program to weekly clinic visit or community based intervention had different age group of children. In present study age up to 36 month was included and mean age of starting therapy was $12.4 \pm 8.6$ months with age range of 3-24 months. Similar age group had been documented by Lakhan et $\mathrm{al}^{[20]}$ in which they enrolled children between 6 to 36 months. In a study by Jin Y Shin et al from Vietnam ${ }^{[3]}$ they had enrolled 46 children in age range of 3-6 years. As per guidelines of IDEA (Individuals with Disabilities 
Education Act) of United States, the intervention services are designed to meet the expectations of developmental needs of children, from birth to three years, who have a delay in physical, cognitive, communicative, social, emotional or adaptive development or have a diagnosed condition that has a high probability of resulting in developmental delay. So to be fruitful during first thousands days or period of plasticity of developing brain, intervention should start at earliest for better outcome.

In present study, $65 \%$ of families belonged to urban set up. Paramleen Kaur et al ${ }^{[21]}$ had shown urban population of $70 \%$ in their study. Lack of awareness is possible reason for the lesser number of patients from villages. Access to comprehensive developmental health services is largely restricted to urban areas. There is felt need to address this issue of availability of early intervention service through community rehabilitation models or training of grass root workers in developing country for better outcome of neurodevelopmental disability.

Family support system is an important component in management of developmental disability and lifelong impairments. Support from family members might directly or indirectly affect adherence to therapy either at home or in clinical settings. In present study $60 \%$ of families were joint family. In a study by Paramleen Kaur et al [21] they had documented $44 \%$ of joint family. Findings from developed countries on this aspect is usually not available as they had assessed parent's education and age but not family structure.

In present study $13 \%$ of father and $20 \%$ of mother were uneducated. In a study by Jin Y Shin ${ }^{[3]}$ none of parents were uneducated and $80 \%$ of parents were educated high school and above. It can be presumed that age of starting therapy, its continuation and adherence to therapy may be dependent on educational level of parents. In present study we did not find any significant correlation between parent's education level and outcome at six month of therapy. This can be possible as if parents are counselled properly, motivated and supported by regular feedback weekly institution based plus daily home therapy can be very useful for bringing improvement in developmental potential of a child with GDD.

In present study it was found that more severe forms of GDD were brought to clinic early for starting therapy. Severe forms of GDD were noted in $42 \%$ of children in present study. In a study by M.-H. Tang et al ${ }^{[2]}$ they had $50 \%$ of children with <-3Sd in form of severe GDD. In a study by Lakhan et al $71 \%$ of children were having moderate, severe or profound developmental delay. In present study no significant correlation was observed between severity of GDD and improvement in developmental quotients at 6 months. So all the children who received weekly clinic based plus regular home therapy benefitted. Similar finding has been observed by Lakhan et $\mathrm{al}^{[20]}$ who demonstrated that all children receiving services benefited from the intervention.

We found significant improvement (Cohen's d $>0.7$ ) for pre- and post-therapy DASII scores in motor and mental scales. Similar findings of improvement were observed by M.-H. Tang et al, Lakhan $\mathrm{R}$ et al and by Jin $\mathrm{Y}$ Shin et al who demonstrated that early intervention and adding home program to weekly institute/ clinic based program can bring significant improvement in all the developmental domains ${ }^{([2],[3],[20])}$ The tests used to assess developmental domains were different in mentioned studies. In a study by Lakhan $\mathrm{R}$ et $\mathrm{al}^{[20]}$ had developed a tool to be used in local language in tribal part of India to assess their child's progress on a Likert scale called Early Intervention Tool (EIT) every three months in the motor, communication, cognitive, and social developmental areas. We have used developmental assessment scale for Indian an infant which is validated, reliable and comprehensive diagnostic scale. In a study by M.H. Tang et $\mathrm{al}^{[2]}$ they had used comprehensive developmental inventory for infants and toddlers (CDIT). 
Present study contributes significantly to the existing scientific body of knowledge of early intervention in which development was assessed by standard valid tool prepared and used in India on DD in a resource-constraint setting.

This study demonstrated the advantages of hybrid method in therapy in form weekly supervised institute based therapy to daily home program. Daily institute based therapy may lead to more drop outs, poor adherence and logistic issues for families and intervention centre when there is discrepancy between strength of therapist and patient load. Home program which are poorly supervised might not give adequate outcome for the benefit of children.

We found modules developed by portage Community based rehabilitation network and NIMH, Secunderabad were easy to understand by parents, simple to practice and monitor. If developmental intervention has to be successful in developing or resource constrained countries intervention tool needs to be easy to practice and requiring less resources. There is need to balance follow up visits at clinic with logistic support family can afford. Present study has shown promising result for "supervised' home therapy program for developmental delay.

\section{Conclusions}

In present study it was observed that home based activity program supervised by weekly institutional visits is highly effective for improvement in developmental outcomes in children with global developmental delay.

\section{Limitations}

We had to drop eighty-one children from study due to non-adherence to follow up so at the end it has less number of children enrolled. This study was not randomized control type of study. We could not carry out detailed cluster based analysis of DASII report which might had given inputs regarding specific domains that needs to be more supervised.

\section{Acknowledgements}

Author is thankful to psychologist, special educator, physiotherapist, social worker and data entry operator of District Early Intervention Centre (DEIC) for helping in various aspect of study like DASII test, preparing goals for therapy, training parents on home based program, task analysis and ensuring compliance to therapy.

\section{Funding: None \\ Conflict of Interest: None}

\section{References}

1. Black M. M., Walker, S. P., Fernald, L. C. H., Andersen, C. T., Digirolamo, A. M., Lu, C., Grantham-Mcgregor, S. (2017). Advancing Early Childhood Development: from Science to Scale 1 Early childhood development coming of age: science through the life course. The Lancet, 389, 77-90. https://doi.org/10.1016/S01406736(16)31389-7

2. Tang MH, Lin CK, Lin WH, Chen CH, Tsai SW, et al. (2011) The effect of adding a home program to weekly institutional-based therapy for children with undefi ned developmental delay: A pilot randomized clinical trial. J Chin Med Assoc 74: 259-266. Link: https://tinyurl.com/y9fl nmoy

3. Shin JY, Nguyen Duc S, Accardo M, Dill CA (2018) The Effects of a Home-Based Intervention for Young Children with Developmental Delays in Vietnam: 6-Month Follow-Up Results. Arch Community Med Public Health 4(1): 017-025. DOI: http://dx.doi.org/10.17352/2455-5479.000035

4. Lozano R, Wang H, Foreman KJ, Rajaratnam JK, Naghavi M, Marcus JR, Murray CJL. Progress towards Millennium Development Goals 4 and 5 on maternal and child mortality: An updated systematic analysis. The Lancet. 2011; 378(9797): 1139-1165

5. Verma RK, Kishore MT. Needs of Indian parents having children with intellectual disability. International Journal of 
Rehabilitation Research. 2009; 32(1):71-76. [PubMed: 19648804]

6. Mc Kenzie K, Milton M, Smith G, OuelletteKuntz H. Systematic review of the prevalence and incidence of intellectual disabilities: current trends and issues. Curr Dev Disord Rep. 2016;3:104-15

7. Dipti kapoor and sharmila B Mukharjee. Global Developmental Delay and Intellectual Disability in Indian Children - Where do we Stand. Indian pediatr; Volume 55 December 15, 2018.1083-85.

8. Dunst CJ, Trivette CM, Hamby DW. Metaanalysis of family-centered help giving practices research. Mental Retarda-tion and Developmental Disabilities Research Reviews. 2007; 13(4):370-378. http://doi.org/10.1002/mrdd.20176. [PubMed: 17979208

9. Kim JM, Mahoney G. The effects of relationship focused intervention on Korean parents and their young children with disabilities. Research In Developmental Disabilities. 2005; 26(2):117-130. [PubMed: 15590243]

10. Agazzi H, Tan SY, Ogg J, Armstrong K, Kirby RS (2017) Does parent-child interaction therapy reduce maternal stress, anxiety, and depression among mothers of children with autism spectrum disorder?. Child Fam Behav Ther 39: 283-303.

11. Wagner SM, McNeil CB (2008) Parent-Child Interaction Therapy for ADHD: A Conceptual Overview and Critical Literature Review. Child Fam Behav Ther 30: 231-256.

12. Eyberg SM, Funderburk BW, Hembree-Kigin TL, McNeil CB, Querido JG, et al. (2001) Parent-child interaction therapy with behavior problem children: One and two year maintenance of treatment effects in the family. Child Fam Behav Ther 23: 1-20.

13. CESA 5 (2003) Portage guide: Birth to six: Activities and routines for pre-schoolers. Portage, Wisconsin: CESA 5.
14. Einfeld SL, Stancliffe RJ, Gray KM, Sofronoff K, Rice L, et al. (2012) Interventions Provided by Parents for Children with Intellectual Disabilities in Low and Middle Income Countries. Journal of Applied Research in Intellectual Disabilities 25: 135-142.

15. Thorburn M J, Odom L, Hanson M, Blackman J, Kaul S (2003) Early Intervention Practices Around the World. Baltimore, lowcost early intervention programs 191-208

16. Dr.Amar Jyothi Persha, N.C.Srinivas, R.C.Nitnaware. Manual on Early Intervention to Infants \& Toddlers with Developmental Delays - Series - 1 (Cognition, Audition \& Vision). Secunderabad. National Institute for the mentally handicapped. 2011

17. Dr.Amar Jyothi Persha, N.C.Srinivas, R.C.Nitnaware. Manual on Early Intervention to Infants \& Toddlers with Developmental Delays - Series - 2 (Gross motor \& Fine motor). Secunderabad. National Institute for the mentally handicapped. 2011

18. Dr.Amar Jyothi Persha, N.C.Srinivas, R.C.Nitnaware Manual on Early Intervention to Infants \& Toddlers with Developmental Delays - Series - 3 (Speech, Language \& Communication). Secunderabad. National Institute for the mentally handicapped. 2011

19. Patni B. (2012). Developmental assessment scale $\mathrm{s}$ for Indian infants (DASII). Indian Journal of practical paediatrics.14.409-12.

20. Lakhan R, Mario A, Qureshi FN, Hall ML. Early intervention services to children with developmental delay in resource poor settings in India. NJMS Volume 02 Number 02| JulyDecember 2013.149-55.

21. Paramleen Kaur, B.S. Chavan, Sneh Lata, Amandeep Kaur, Sophia Tinku, Yashwant Arora and Vani Ratnam. Early Intervention in Developmental Delay. Indian Journal of Pediatrics, Volume 73; May, 2006. 405-08. 